\title{
Automatic Initialization Algorithm for Carotid Artery Segmentation in CTA Images
}

\author{
Martijn Sanderse ${ }^{1}$, Henk A. Marquering ${ }^{1}$, Emile A. Hendriks ${ }^{2}$, \\ Aad van der $\operatorname{Lugt}^{3}$, and Johan H.C. Reiber ${ }^{1}$
}

1 Dept. of Radiology, Div. of Image Processing, LUMC, Leiden, The Netherlands

\{M.Sanderse, H.A.Marquering, J.H.C.Reiber\}@lumc.nl

2 ICT Group, Delft Univ. of Technology, Delft, The Netherlands

\author{
E.A.Hendriks@ewi.tudelft.nl
}

3 Dept. Of Radiology, Erasmus Medical Center, Rotterdam, The Netherlands

A. vanderLugt@erasmusmc.nl

\begin{abstract}
Analysis of CT datasets is commonly time consuming because of the required manual interaction. We present a novel and fast automatic initialization algorithm to detect the carotid arteries providing a fully automated approach of the segmentation and centerline detection. First, the volume of interest (VOI) is estimated using a shoulder landmark. The carotid arteries are subsequently detected in axial slices of the VOI by applying a circular Hough transform. To select carotid arteries related signals in the Hough space, a 3-D, direction dependent hierarchical clustering is used. To allow a successful detection for a wide range of vessel diameters, a feedback architecture was introduced. The algorithm was designed and optimized using a training set of 20 patients and subsequently evaluated using 31 test datasets. The detection algorithm, including VOI estimation, correctly detects $88 \%$ of the carotid arteries. Even though not all carotid arteries have been correctly detected, the results are very promising.
\end{abstract}

\section{Introduction}

CT angiography images an anatomical region as a $3 \mathrm{D}$ volume. The axial source images can be used for visual inspection and to grade the severity of stenosis. However, this is a tedious job and it does not make full use of the advantages of $3 \mathrm{D}$ visualization. The $3 \mathrm{D}$ volume representation offers several post processing techniques such as Curved Planar Reformation (CPR) [1 to ease the evaluation of the artery. CPR resamples the dataset such that the centerline of an artery becomes a straight line. In current visualization workstations centerlines are not detected automatically, and user interaction is required to position several points inside the vessel [e.g. Vessel View; Siemens, Forchheim, Germany]. Several segmentation techniques and centerline detection algorithms can be used to segment the carotid arteries and detect their centerline 2 345]. However, all of these techniques need to be initialized manually by the specification of the start and end points of the artery segment. 
Here we present an Automatic iNITialization Algorithm (AnitA) to select the carotid arteries providing a fully automated approach of the segmentation of the carotid arteries and the detection of its centerline. This algorithm excludes any user interaction and therefore reduces the time required for the analysis of carotid arteries. The algorithm is designed to position a single initialization point in the common carotid arteries. This point can then be used by common segmentation algorithms, such as a wave front propagation segmentation algorithm [6]. Usually a full examination of the carotid arteries using CT images takes approximately 30 to 45 minutes. It is expected that a full automatic segmentation can reduce this time to 5 to 10 minutes.

There are only a few papers known that are concerned with the fully automatic detection of arteries. Cline et al. 7] have developed a method to automatically segment the coronary vessel tree with mathematical morphology operations. Lorenz [8] describes a heuristic approach for delineation of the cardiac volume, which includes the automatic detection of the descending aorta. Both methods are heuristic methods for different vessels and therefore not suitable to adapt to carotid artery detection.

To our knowledge no literature has been published that describes a fast and fully automated segmentation of a peripheral vessel (segment) in 3D datasets. Here we present a fast initialization algorithm that allows the fully automatic start of a the segmentation of the carotid arteries, which may be generally applicable to other vessels.

\section{Methods}

AnitA consist of two main steps. Because the lower part of the neck is best suited to detect the common carotid arteries, this area, or volume of interest (VOI) is estimated first. In the second step the common carotid arteries are detected in the VOI and the initialization point is selected for each carotid artery.

To automatically determine the VOI it is desirable to make use of distinct image features, or landmarks. A distinct (spatial) feature in CT images is the (vertical) position of the shoulders, which is close to the common carotid arteries. The shoulders are detected in Maximum Intensity Projections (MIPs) of the dataset on the coronal plane (xz-plane). The detection process starts at two positions: $6 \mathrm{~cm}$ left and right of the center of the MIP (figure 1). The closests pixels with bone intensities below these starting points are considered to be the shoulder landmark.

The VOI boundaries can be estimated based upon this position. First the VOI boundaries are manually determined in the training datasets. From the distances between these positions in single datasets, a general relation between VOI boundaries and landmarks is determined.

Because the common carotid arteries have a tubular shape and run near vertically, their appearance in horizontal slices approximates circles. The carotid arteries are detected in a number of steps, which are described below. First, small size contributions are removed by smoothing the image using a convolution with 


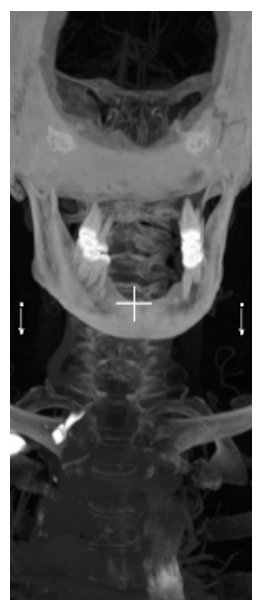

Fig. 1. Landmark detection starts next to the center of the MIP, and is then directed downwards

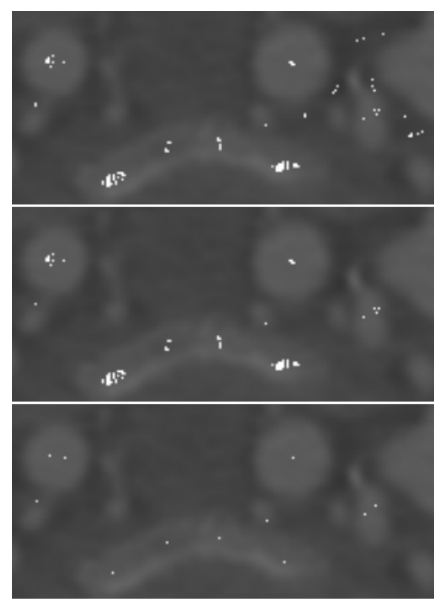

Fig. 2. Maxima (white dots) in a slice after thresholding Hough space (top), maxima reduced by threshold of values in corresponding position in original image (middle), and a second reduction by clustering and replacing the cluster with its center of gravity (bottom)

a Gaussian. In order to detect circles in the images, we need to extract the contour of the carotid arteries. Edges are detected by using a combination of the gradient magnitude and the Laplacian of the image in horizontal slices. Edges that are typical for the carotid arteries are selected by thresholding the gradient magnitude, excluding edges that are too sharp or too soft. The edge image is subsequently used to detect potential center points of the carotid arteries using the circular Hough transform. The Hough transform can be expressed as a convolution operation, using a circle with a radius $r$ as the kernel:

$$
\begin{gathered}
H=E \otimes K_{\text {Circle }} \\
K_{\text {Circle }}=\left\{\begin{array}{lc}
1, & \text { iff } \\
0, & \text { otherwise }
\end{array} m^{2}+n^{2}=r^{2}\right.
\end{gathered}
$$

with $H$ the Hough response, $E$ the binary edge image, and $K_{C i r c l e}$ the circle kernel. The pixels that are most likely to be center points of a circle appear as maxima in the output image of the convolution operation. In order to be able to detect circles for a range of radii in one convolution operation, the kernel can be shaped like an annulus:

$$
K_{\text {Annulus }}=\left\{\begin{array}{lc}
1, & \text { iff } \\
0, & \text { otherwise }
\end{array} \quad r_{\min }^{2}<m^{2}+n^{2}<r_{\max }^{2}\right.
$$


with $K_{\text {Annulus }}$ the annulus kernel, and $r_{\min }$ and $r_{\max }$ the minimum and maximum radius of the annulus 9 . To remove the contributions that are not related to the carotid arteries, only the local maxima are selected that have an intensity of at least $90 \%$ of the maximum intensity (figure 2). To reduce the number of maxima even further, the intensity in the original image at the corresponding positions is used to discard maxima that do not have an intensity value that is typical for contrast-enhanced blood. Because in a single slice multiple maxima per carotid artery are found, the number of maxima is finally reduced by clustering these maxima per slice. This can reduce the number of maxima per carotid artery to one, in a single slice. The clustering technique also reduces the number of non-carotid related maxima.

The procedure described above, produces a $3 \mathrm{D}$ volume with center points that are potentially related to the carotid arteries. To distinguish the carotid artery center points from other objects, we can make use of knowledge on the tubular shape and the running of the carotid arteries. This near vertical running of the common carotid arteries results in little deviation in horizontal position in subsequent axial slices. To exploit this anatomical property, a 3D clustering is applied in which a point is added to a cluster if the distance to the cluster is not larger than a given direction dependent distance. The distance between a cluster and a point is defined as the minimum distance between the point and any other point of the cluster and is calculated as

$$
d=\sqrt{x^{2}+y^{2}+(\alpha \cdot z)^{2}}, \quad \text { with } 0<\alpha<1
$$

where $\alpha$ allows for larger deviation in the $z$-direction and consequently puts restrictions on the horizontal distance in the clustering. The two clusters containing the most Hough maxima are considered to contain the center points of the left and right common carotid artery.

A self test was introduced to value the results from the detection process based upon the number of center points per cluster. If the number of center points in one of the clusters was below a threshold, the detection process can be repeated using a different value for the annulus kernel. After the second run, the self test is used again to determine if the smaller radius range annulus provides a satisfying detection.

Finally, from each cluster the point with the highest intensity in Hough space is selected as the final initialization point.

\section{Results}

The estimation of the VOI and the detection of carotids were initially developed using a dataset of 20 patients. For the testing of the algorithm data from another 31 patients were used. This test set consisted of datas from 29 successive patients from one clinic, and two other datasets from two different clinics.

The shoulder landmarks were detected correctly on all test datasets. The VOI detector was constructed by comparing the data from the VOIs determined 
manually and the using the landmarks. The VOI detector based on the shoulder was constructed as follows:

$$
\begin{gathered}
V O I_{\text {upper }}=z_{\text {shoulder }}+d_{V u} \\
V O I_{\text {lower }}=z_{\text {shoulder }}-d_{V l}
\end{gathered}
$$

with $z_{\text {shoulder }}$ the position of the shoulder landmark in the VOI, and $d_{V u}$ and $d_{V l}$ the average distance between upper and lower VOI boundaries and shoulder landmark. Table 1 and figure 3 show the results of the VOI estimation.

Because the carotid artery must be detected in a number of subsequent slices, the VOI does not have to be estimated perfectly accurate. However, it is important that the common carotid arteries are present in the bulk of the slices of the VOI. Therefore the VOIs are evaluated on the basis of the percentage of the slices in which the common carotid arteries are present. Taken the carotid artery detection in consideration, the VOI detection is considered successful if the common carotid arteries are present in at least $70 \%$ of the slices. For all steps of the carotid artery detection in the VOI, initially a range of values was used for each parameter to inspect which values gave the best performance. Because the optimal values of some parameters are dependent on the settings of other parameters, continuous tuning of the values of all parameters was done while developing the algorithm. Final values for all parameters can be found in Table 2 .

On average the Hough transform resulted in 50 maxima for each slice. After removing contributions that do not have typical values for contrast enhanced blood in the original image in corresponding positions, on average 40 maxima

Table 1. VOI estimation results

\begin{tabular}{ll}
\hline & Number of test datasets \\
\hline Total number of datasets tested & 31 \\
$100 \%$ common carotid arteries present in slices & 23 \\
$\geq 70 \%$ common carotid arteries present in slices & 30 \\
$\geq 50 \%$ common carotid arteries present in slices & 31 \\
\hline
\end{tabular}

Table 2. Values of parameters for carotid arteries detection

\begin{tabular}{ll}
\hline Standard deviation Gaussian for smoothing & $1.8 \mathrm{~mm}$ \\
Zero crossing margin in edge detector & $10 \mathrm{HU} / \mathrm{mm}^{2}$ \\
Maximum gradient magnitude in edge detector & $860 \mathrm{HU} / \mathrm{mm}$ \\
Minimum gradient magnitude in edge detector & $172 \mathrm{HU} / \mathrm{mm}$ \\
Maximum clustering distance in 2D in maxima reduction & $5.8 \mathrm{~mm}$ \\
Maximum cluster distance in 3D & $5.8 \mathrm{~mm}$ \\
Factor to allow large cluster distance in z-direction $\alpha$ & 0.001 \\
First Hough transform annulus kernel radius-range & $3.5 \mathrm{~mm}<r<4.5 \mathrm{~mm}$ \\
Second Hough transform annulus kernel radius-range & $2.5 \mathrm{~mm}<r<3.5 \mathrm{~mm}$ \\
\hline
\end{tabular}


were present in each slice. After the reduction using the $2 \mathrm{D}$ clustering only 10 maxima were present on average in each slice. For the evaluation of the presented method, the carotid artery detection was performed on the test dataset. The algorithm provided 50 initialization points (25 datasets) that were considered accurate according to the self evaluation test. Of these 25 datasets, 16 were detected during the initial run and in 9 datasets the carotid arteries were detected in the second run. In 6 datasets, the self evaluation test decided that no accurate carotid artery detection was performed. Subsequently, the results of all test datasets were inspected visually. Initialization points that were classified as successful by the self test and proved to be correct by visual inspection were labeled true positive, see table 3 . All true positive points were usable as initialization points according to expert opinion. In the training set all datasets were classified as successful by the self test. In the first run 37 initialization points were correctly positioned, after a second run, on three datasets, 39 initialization points were positioned correctly in total, resulting in a true positive percentage

Table 3. Results from carotid artery detection in estimated VOIs in test datasets

\begin{tabular}{ll}
\hline True positive & $91 \%$ \\
True negative & $67 \%$ \\
Unsuccessful datasets after re-run & $21 \%$ \\
\hline
\end{tabular}

Table 4. Total numbers of correct and uncorrect detected initialization points in training and test sets

\begin{tabular}{llll}
\hline & \# Correct init. points & \# False init. points & Percentage \\
\hline Training set (20 datasets) & 39 & 1 & $97.5 \%$ \\
Test set (31 datasets) & 51 & 11 & $82.3 \%$ \\
Total (51 datasets) & 90 & 12 & $88.2 \%$ \\
\hline
\end{tabular}

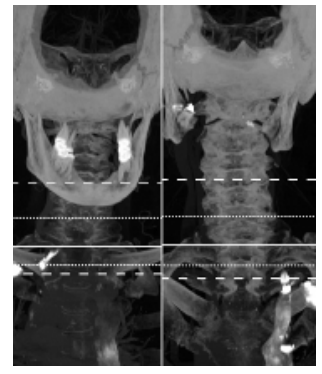

Fig. 3. Two VOI estimations, shoulder landmark in solid line, estimated VOI in dotted lines, manually selected VOI in dashed lines

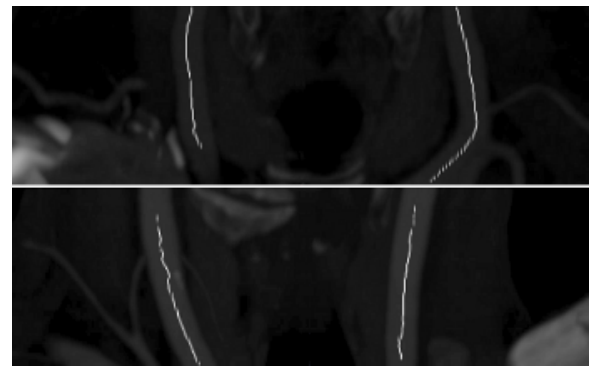

Fig. 4. Slab based MIPs of two VOIs, showing all center points found in the two largest clusters. They are positioned inside the carotid arteries. 
of $97.5 \%$. Table 4 shows the total numbers of correct and incorrect initialization points according to expert opinion. Figure 4 shows the detected centerpoints of the carotid arteries in slab based MIPs.

The average time necessary to detect the starting points in the datasets, including the time needed to read the data from hard disk is $120 \mathrm{sec}$. with a standard deviation of 20 sec., on a Pentium IV 2.5Ghz processor, with 1 GB of memory.

\section{Discussion}

We have presented a full automatic approach of detection the carotid arteries in CTA datasets using a VOI estimation and a Hough space based carotid artery detection. The shoulder landmark was detected correctly in all datasets, resulting in accurate VOI estimations using the average distance of shoulder and VOI boundaries.

While testing for the optimal radius for the Hough transform, most carotid arteries were detected using a radius of approximately $4 \mathrm{~mm}$. In some datasets $4 \mathrm{~mm}$ was too large and a radius of around $3 \mathrm{~mm}$ gave correct results. It was not possible to detect carotid arteries using a Hough annulus kernel with a radius range that included both radii, because too many contributions from other structures in the Hough space were present. This was solved by introducing a feedback architecture using the self test allowing two runs with a different radius range. The self test was also used to grade the performance of the carotid artery detection. Currently this use of the test results in too many false negatives. Improvement of the self test is expected by incorporating a more advanced analysis of the shape and position of the cluster and anatomical knowledge.

The carotid artery detection was unsuccessful for a few datasets, because the intensity of the maxima related to the carotid arteries was smaller than required. Careful inspection of the various steps indicated that this can happen when parts of the contour are not detected. In some datasets the carotid arteries were detected, but in too few slices, resulting in a rejection by the self test classifier. Another cause for failure was the strong variation in the density of contrast material in CT images. In these cases, the intensities in other arteries and veins was significantly higher than was observed in the training set. As a result of this, in one dataset a jugular vein was detected instead of carotid arteries in the test set. It is expected that a full automatic segmentation can reduce analysis time of a single dataset from 30-45 minutes to 5-10 minutes. It has been shown that AnitA can detect the carotid arteries on average in $88 \%$, therefore this approach can reduce the analysis time by a factor 3 on average.

\section{Conclusions}

Currently, a significant amount of time in the evaluation of arteries in CTA images is concerned with the manual interaction for the centerline detection. We have presented a novel approach for the full automatic detection of the 
carotid arteries. The implementation of such an automated detection results in a significant reduction of analysis time of the carotid arteries in CT-images.

The combination of the VOI estimator based on the shoulder landmark and a carotid artery detection based on a circular Hough transform and a hierarchical clustering algorithm has been proved to be successful on 31 test datasets. In $97 \%$ the VOI detection is correct, and in $88 \%$ the carotid artery detection in the estimated VOIs is correct. Even though not all carotid artery detections were successful, the results are very promising.

\section{References}

1. Kanitsar, A., Fleischmann, D., Wegenkittl, R., Felkel, P., Gröller, M.E.: Cpr: curved planar reformation. In: VIS '02: Proceedings of the conference on Visualization '02, Washington, DC, USA, IEEE Computer Society (2002) 37-44

2. Felkel, P., Wegenkittl, R., Kanitsar, A.: Vessel tracking in peripheral cta datasets an overview. In: SCCG '01: Proceedings of the 17th Spring conference on Computer graphics, Washington, DC, USA, IEEE Computer Society (2001) 232

3. Kirbas, C., Quek, F.: A review of vessel extraction techniques and algorithms. ACM Comput. Surv. 36 (2004) 81-121

4. van Andl, H.A.F.G., Meijering, E.H.W., van der Lugt, A., Vrooman, H.A., Stokking, R.: Vampire: Improved method for automated center lumen line definition in atherosclerotic carotid arteries in cta data. In: MICCAI (1). (2004) 525-532

5. Koning, P.D.: Automated segmentation and analysis of vascular structures in magnetic resonance images. Magnetic Resonance Images 50 (2003) 1189-1198

6. Marquering, H.A., Dijkstra, J., Koning, P.J.D., Stoel, B.C., Reiber, J.H.: Towards quantitative analysis of coronary cta. The International Journal of Cardiovascular Imaging 21 (2005) 73-84

7. Cline, H.E., Thedens, D., Irarrazaval, P., Meyer, C., Hu, B., Nishimura, D., Ludke, S.: 3d mr coronary artery segmentation. Magnetic Resonance in Medicine 40 (1998) 697-702

8. Lorenz, C., Lessick, J., Lavi, G., Bülow, T., Renisch, S.: Fast automatic delineation of cardiac volume of interest in msct images. In: Medical Imaging 2004: Image Processing. Edited by Fitzpatrick, J. Michael; Sonka, Milan. Proceedings of the SPIE. Volume 5370. (2004) 456-466

9. Kerbyson, D., Atherton, T.: Circle detection using hough transform filters. In: Image Processing and its Applications, Fifth International Conference on. (1995) $370-374$ 